\title{
Fuzzy version of Meir-Keeler type contractive condition and existence of fixed point
}

\author{
Saurabh Manro ${ }^{a, *}$, Sanjay Kumar ${ }^{b}$, S. S. Bhatia ${ }^{a}$ \\ ${ }^{a}$ School of Mathematics and Computer Applications, Thapar University, Patiala, Punjab, India \\ ${ }^{b}$ Deenbandhu Chhotu Ram University of Science and Technology, Murthal, Sonepat, India \\ * Corresponding Author \\ E-mail: sauravmanro@hotmail.com
}

\begin{abstract}
In this article, we prove a general common fixed point theorem for two pairs of weakly compatible self-mappings of a fuzzy metric space satisfying a generalized Meir-Keeler type contractive condition. Our results substantially extend, generalize, improve and fuzzify multitude of well known results of the form existing in literature in metric as well as fuzzy metric spaces.
\end{abstract}

2000 Mathematics Subject Classification. 47H10. 54H25.

Keywords. Weakly compatible maps, fuzzy metric space, common property (E.A), JCLR $R_{S T}$ property, Meir-Keeler type contractive condition.

\section{Introduction}

Meir and Keeler in [18] considered an extension of the classical Banach contraction theorem on a complete metric space. The concept of fuzzy sets was introduced by Zadeh [29] in 1965. In 1975, Kramosil and Michalek [15] gave the notion of fuzzy metric spaces which could be considered as a reformulation, in the fuzzy context, of the notion of probabilistic metric space due to Menger [19]. On the other hand, Fixed point theorems give the conditions under which maps (single or multivalued) have solutions. The theory itself is a beautiful mixture of analysis (pure and applied), topology, and geometry. Over the last 50 years or so the theory of fixed points has been revealed as a very powerful and important tool in the study of nonlinear phenomena. In particular fixed point techniques have been applied in such diverse fields as biology, chemistry, economics, engineering, game theory and physics. Fixed point theory is one of the most famous mathematical theories with application in several branches of science. Fixed point theory in fuzzy metric spaces has been developed starting with the work of Heilpern [11]. He introduced the concept of fuzzy contraction mappings and proved some fixed point theorems for fuzzy contraction mappings in metric linear spaces, which is a fuzzy extension of the Banach contraction principle. In [8, 9], George and Veeramani introduced and studied the notion of fuzzy metric spaces which constitutes a modification of the one due to Kramosil and Michalek. From now on, by fuzzy metric we mean a fuzzy metric in the sense of George and Veeramani. Many authors have contributed to the development of this theory and apply to fixed point theory, for instance $[1,4,6,7,12,16,17,20-24$, $26,28]$.

In 1976, Jungck [13] introduced the notion of commuting mappings. Afterward, Sessa [27] gave the notion of weakly commuting mappings. Jungck [14] defined the notion of compatible mappings to generalize the concept of weak commutativity and showed that weakly commuting mappings are compatible but the converse is not true. The concept of property $(E . A)$ in metric space has been recently introduced by Aamri and El Moutawakil [3]. The concept of property (E.A) allows 
to replace the completeness requirement of the space with a more natural condition of closeness of the range. In 2009, M. Abbas et al.[3] introduced the notion of common property (E.A).

Recently in 2012, Chauhan et al.[5] introduced the concept of $J C L R_{S T}$ property and also established existence of a common fixed point theorems for generalize contractive mappings satisfy this property in fuzzy metric spaces.

The aim of this work is to introduce generalized Meir-Keeler type contractive condition in fuzzy metric space. Moreover, we establish some new existence of a common fixed point theorem for generalized contractive mappings in fuzzy metric spaces by using generalized Meir-Keeler type contractive condition and give some examples. Our theorems generalize, unify, and extend many results in literature.

\section{Preliminaries}

The concept of triangular norms ( $t$-norms) is originally introduced by Menger [23] in study of statistical metric spaces.

Definition 2.1. [33] A binary operation $*:[0,1] \times[0,1] \rightarrow[0,1]$ is said to be a continuous $t$-norm if $*$ satisfies the following conditions:

(i) $*$ is commutative and associative;

$(i i) *$ is continuous;

(iii) $a * 1=a$ for all $a \in[0,1]$

(iv) $a * b \leq c * d$ whenever $a \leq c$ and $b \leq d$ for all $a, b, c, d \in[0,1]$.

Examples of $t$-norms are $a * b=\min \{a, b\}, a * b=a . b$ and $a * b=\max \{a+b-1,0\}$.

Definition 2.2. [1] A 3-tuple $(X, M, *)$ is said to be a fuzzy metric space if $X$ is an arbitrary set, $*$ is a continuous $t$-norm and $M$ is a fuzzy set on $X^{2} \times[0, \infty)$ satisfying the following conditions:for all $x, y, z \in X$ and $s, t>0$,

(i) $M(x, y, 0)=0$;

(ii) $M(x, y, t)=1$ if and only if $x=y$;

(iii) $M(x, y, t)=M(y, x, t)$;

(iv) $M(x, y, t) * M(y, z, s) \leq M(x, z, t+s)$;

(v) $M(x, y,):.[0, \infty) \rightarrow[0,1]$ is left continuous

The function $M(x, y, t)$ denotes the degree of nearness between $x$ and $y$ w.r.t. $t$ respectively.

Remark 2.3. In fuzzy metric space $(X, M, *), M(x, y,$.$) is non-decreasing for all x, y \in X$.

Definition 2.4. [1] Let $(X, M, *)$ be a fuzzy metric space. Then a sequence $\left\{x_{n}\right\}$ in $X$ is said to be 
(i) a Cauchy sequence if, for all $t>0$ and $p>0, \lim _{n \rightarrow \infty} M\left(x_{n+p}, x_{n}, t\right)=1$;

(ii) convergent to a point $x \in X$ if, for all $t>0, \lim _{n \rightarrow \infty} M\left(x_{n}, x, t\right)=1$.

Definition 2.5. [1] A fuzzy metric space $(X, M, *)$ is said to be complete if and only if every Cauchy sequence in $X$ is convergent.

Example 2.6. [1] Let $X=\left\{\frac{1}{n}: n \in N\right\} \cup\{0\}$ and let $*$ be the continuous $t$-norm defined by $a * b=$ $a . b$ for all $a, b \in[0,1]$. For each $t>0$ and $x, y \in X$, define $(X, M, *)$ by $M(x, y, t)=\frac{t}{t+|x-y|}, t>0$ and $M(x, y, 0)=0$. Clearly, $(X, M, *)$ is a complete fuzzy metric space.

Definition 2.7. [1] A pair of self mappings $(f, g)$ of a fuzzy metric space $(X, M, *)$ is said to be compatible if $\lim _{n \rightarrow \infty} M\left(f g x_{n}, g f x_{n}, t\right)=1$ whenever $\left\{x_{n}\right\}$ is a sequence in $X$ such that

$$
\lim _{n \rightarrow \infty} f x_{n}=\lim _{n \rightarrow \infty} g x_{n}=z
$$

for some $z \in X$.

Definition 2.8. [1] Two self mappings $f$ and $g$ of an fuzzy metric space $(X, M, *)$ are called non-compatible if there exists at least one sequence $\left\{x_{n}\right\}$ in $X$ such that

$$
\lim _{n \rightarrow \infty} f x_{n}=\lim _{n \rightarrow \infty} g x_{n}=z
$$

for some $z \in X$ but either $\lim _{n \rightarrow \infty} M\left(f g x_{n}, g f x_{n}, t\right) \neq 1$ or the limit does not exist.

Definition 2.9. [13] Two self-mappings $f$ and $g$ of a nonempty set $X$ are said to be weakly compatible if $f g x=g f x$ for all $x$ at which $f x=g x$.

Definition 2.10. [4] A pair of self-mappings $(f, g)$ on a fuzzy metric space $(X, M, *)$ is said to satisfy the property $(E . A)$ if there exists a sequence $\left\{x_{n}\right\}$ in $X$ such that

$$
\lim _{n \rightarrow \infty} f x_{n}=\lim _{n \rightarrow \infty} g x_{n}=z
$$

for some $z \in X$.

The class of E.A. mappings contains the class of non compatible mappings.

Definition 2.11. [15] The pairs $(A, S)$ and $(B, T)$ on a fuzzy metric space $(X, M, *)$ are said to satisfy the common property $(E . A)$ if there exist two sequences $\left\{x_{n}\right\}$ and $\left\{y_{n}\right\}$ in $X$ such that

$$
\lim _{n \rightarrow \infty} A x_{n}=\lim _{n \rightarrow \infty} S x_{n}=\lim _{n \rightarrow \infty} B y_{n}=\lim _{n \rightarrow \infty} T y_{n}=z
$$

for some $z \in X$.

In 2012, Chauhan et al. [6] introduced the concept of the $J C L R_{S T}$ property as follows:

Definition 2.12. [6] The pairs $(A, S)$ and $(B, T)$ on a fuzzy metric space $(X, M, *)$ are said to satisfy the $J C L R_{S T}$ property if there exist two sequences $\left\{x_{n}\right\}$ and $\left\{y_{n}\right\}$ in $X$ such that

$$
\lim _{n \rightarrow \infty} A x_{n}=\lim _{n \rightarrow \infty} S x_{n}=\lim _{n \rightarrow \infty} B y_{n}=\lim _{n \rightarrow \infty} T y_{n}=S z=T z
$$

for some $z \in X$. 
Example 2.13. Let $(X, M, *)$ be a fuzzy metric space with $X=[-1,1]$ and for all $x, y \in X$ by $M(x, y, t)=\frac{t}{t+|x-y|}, t>0$ and $M(x, y, 0)=0$ where $a * b=\min \{a, b\}$ for all $a, b \in[0,1]$ respectively. Define self maps $A, B, S$ and $T$ on $X$ as $A x=\frac{x}{3}, B x=\frac{-x}{3}, S x=x, T x=-x$ for all $x \in X$. Then, with sequences $\left\{x_{n}\right\}=\left\{\frac{1}{n}\right\}$, and $\left\{y_{n}\right\}=\left\{\frac{-1}{n}\right\}$, in $X$, one can easily verify that

$$
\lim _{n \rightarrow \infty} A x_{n}=\lim _{n \rightarrow \infty} S x_{n}=\lim _{n \rightarrow \infty} B y_{n}=\lim _{n \rightarrow \infty} T y_{n}=S(0)=T(0)
$$

. This shows that the pairs $(A, S)$ and $(B, T)$ satisfy $J C L R_{S T}$ property.

\section{$3 \quad$ Main Results}

Theorem 3.1. Let $A, B, S$ and $T$ be four self maps of a fuzzy metric space $(X, M, *)$, with $a * b$ $=\min \{a, b\}$ for all $a, b \in[0,1]$, such that

(3.1) $A X \subseteq T X$ and $B X \subseteq S X$;

(3.2) given an $\varepsilon>0$ and for all $x, y \in X$, there exists a $\delta \in(0, \varepsilon)$ such that

$\varepsilon-\delta<m(x, y, t) \leq \varepsilon \Rightarrow M(A x, B y, t)>\varepsilon, \ldots(1)$

where $m(x, y, t)=\min \{M(S x, T y, t), M(A x, S x, t), M(B y, T y, t)\}$;

(3.3) one of $A X, B X, S X$ or $T X$ is a complete subspace of $X$.

Then

(I) $A$ and $S$ have a coincidence point,

(II) $B$ and $T$ have a coincidence point.

Moreover, if the pair $(A, S)$, as well as $(B, T)$, are weakly compatible, then the maps $A, B, S$ and $T$ have a unique common fixed point in $X$.

Proof. Let $x_{0}$ be an arbitrary point in $X$. Define sequences $\left\{x_{n}\right\}$ and $\left\{y_{n}\right\}$ in $X$ by using (3.1),

$$
y_{2 n-1}=T x_{2 n-1}=A x_{2 n-2}
$$

and

$$
y_{2 n}=S x_{2 n}=B x_{2 n-1} \ldots . .(2)
$$

We claim that $\left\{y_{n}\right\}$ is a Cauchy sequence. Let $M_{n}=M\left(y_{n}, y_{n+1}, t\right)$ and $N_{n}=N\left(y_{n}, y_{n+1}, t\right)$ where $t>0$.

Two cases arises. Suppose that $M_{n}=1$ for some $n=2 k-1$. Then $M\left(y_{2 k-1}, y_{2 k}, t\right)=1$. This gives $y_{2 k-1}=y_{2 k}$, which implies that $T x_{2 k-1}=A x_{2 k-2}=S x_{2 k}=B x_{2 k-1}$, so $T$ and $B$ have a coincidence point. Further, if $M_{n}=1$ for some $n=2 k$, then $M\left(y_{2 k}, y_{2 k+1}, t\right)=1$. This gives $y_{2 k}=y_{2 k+1}$, which implies that $T x_{2 k+1}=A x_{2 k}=S x_{2 k}=B x_{2 k-1}$, so $A$ and $S$ have a coincidence point.

Now suppose that $M_{n} \neq 1$ for all $n$.

If, for some $x, y \in X, m(x, y, t)=1$ then we get $A x=S x$ and $B y=T y$. Hence the result.

If $m(x, y, t)<1$ for all $x, y \in X$, then, by (1), we have

$$
M(A x, B y, t)>m(x, y, t) .
$$


Hence, we have

$M_{2 n-1}=M\left(y_{2 n-1}, y_{2 n}, t\right)=M\left(A x_{2 n-2}, B x_{2 n-1}, t\right)$

$>m\left(x_{2 n-2}, x_{2 n-1}, t\right)$

$=\min \left\{M\left(S x_{2 n-2}, T x_{2 n-1}, t\right), M\left(A x_{2 n-2}, S x_{2 n-2}, t\right), M\left(B x_{2 n-1}, T x_{2 n-1}, t\right)\right\}$

$=\min \left\{M\left(y_{2 n-2}, y_{2 n-1}, t\right), M\left(y_{2 n-1}, y_{2 n-2}, t\right), M\left(y_{2 n}, y_{2 n-1}, t\right)\right\}$

$=\min \left\{M_{2 n-2}, M_{2 n-1}\right\}=M_{2 n-2}$.

Therefore, $M_{2 n-1}>M_{2 n-2} \ldots .(4)$

Similarly, $M_{2 n}>M_{2 n-1}$.

Hence we deduce that $M_{n}>M_{n-1}$ for all $n$.

Thus $\left\{M_{n}\right\}$ is a strictly increasing sequence of positive real numbers in $[0,1]$. Hence, $\left\{M_{n}\right\}$ converges to some limit, say $p . . .(5)$

Next we claim that $p=1$. If $p \neq 1$, then by (5), there exists a $\delta>0$ and a natural number $m$ such that, for each $n \geq m$,

$$
p-\delta<M\left(y_{n}, y_{n+1}, t\right)=M_{n} \leq p
$$

In particular, $m\left(x_{2 n}, x_{2 n-1}, t\right)=\min \left\{M_{2 n}, M_{2 n-1}\right\}=M_{2 n-1}$, and we get $p-\delta<M_{2 n-1} \leq p$. Therefore, by using (1),

$$
M\left(A x_{2 n}, B x_{2 n-1}, t\right)=M\left(y_{2 n+1}, y_{2 n}, t\right)=M_{2 n}>p,
$$

a contradiction. Hence $p=1$; i.e.,

$$
\lim _{n \rightarrow \infty} M_{n}=\lim _{n \rightarrow \infty} M\left(y_{n}, y_{n+1}, t\right)=1 .
$$

Now, for any positive integer $k$,

$$
M\left(y_{n}, y_{n+k}, t\right) \geq M\left(y_{n}, y_{n+1}, \frac{t}{k}\right) * M\left(y_{n+1}, y_{n+2}, \frac{t}{k}\right) * \ldots * M\left(y_{n+k-1}, y_{n+k}, \frac{t}{k}\right) .
$$

Since, $\lim _{n \rightarrow \infty} M\left(y_{n}, y_{n+1}, t\right)=1$ for $t>0$, it follows that

$$
\lim _{n \rightarrow \infty} M\left(y_{n}, y_{n+p}, t\right) \geq 1 * 1 * \ldots * 1=1,
$$

which shows that $\left\{y_{n}\right\}$ is a Cauchy sequence in $X$.

Now suppose that $S X$ is a complete subspace of $X$. Then the subsequence $y_{2 n}=S x_{2 n}$ must have a limit in $S X$, call it $z$, and $v \in S^{-1}(z)$, so that $S v=z$. As $\left\{y_{n}\right\}$ is a Cauchy sequence containing a convergent subsequence $\left\{y_{2 n}\right\}$, the sequence $\left\{y_{n}\right\}$ also converges to $z$.

First we claim that $A v=z$. Suppose not. Then, on setting $x=v$ and $y=x_{2 n-1}$ in (3), one gets, for $t>0$,

$M\left(A v, B x_{2 n-1}, t\right)>m\left(v, x_{2 n-1}, t\right)=\min \left\{M\left(S v, T x_{2 n-1}, t\right), M(S v, A v, t), M\left(B x_{2 n-1}, T x_{2 n-1}, t\right)\right\}$.

Taking the limit as $n \rightarrow \infty$, we have

$$
M(A v, z, t)>\min \{M(z, z, t), M(z, A v, t), M(z, z, t)\}=M(z, A v, t)
$$

a contradiction. Therefore, $A v=z=S v$. Hence the pair $(A, S)$ has a point of coincidence. As $A X \subseteq T X, A v=z$ implies that $z \in T X$. Let $w \in T^{-1}(z)$, then $T w=z$. 
Next, we claim that $B w=z$. Suppose not. Again by using (3), we get

$$
\begin{aligned}
& M\left(y_{2 n+1}, B w, t\right)=M\left(A y_{2 n}, B w, t\right)>m\left(y_{2 n}, w, t\right) \\
& =\min \left\{M\left(S y_{2 n}, T w, t\right), M\left(S y_{2 n}, A y_{2 n}, t\right), M(B w, T w, t)\right\} .
\end{aligned}
$$

Taking the limit as $n \rightarrow \infty$, we have

$$
M(z, B w, t)>\min \{M(z, z, t), M(z, z, t), M(B w, z, t)\}=M(B w, z, t),
$$

a contradiction. Therefore, $B w=z=T w$. Thus the pair $(B, T)$ has a point of coincidence. Hence, we have shown that $z=S v=A v=B w=T w$.

The same result is obtained if we assume $T X$ to be complete. Indeed, if $A X$ is complete, then $z \in A X \subseteq T X$ and if $B X$ is complete, then $z \in B X \subseteq S X$. As the pairs $(A, S)$ and $(B, T)$ are weakly compatible, then $A z=A S v=S A v=S z$ and $B z=B T w=T B w=T z$. Next, we claim that $A z=z$. If not, then by (3), we have

$M(A z, z, t)=M(A z, B w, t)>m(z, w, t)$

$=\min \{M(S z, T w, t), M(S z, A z, t), M(B w, T w, t)\}$

$=\min \{M(A z, z, t), M(A z, A z, t), M(z, z, t)\}=M(A z, z, t)$,

a contradiction. Therefore, $A z=z$. Similarly, one can easily show that $B z=z$. Thus $z$ is unique common fixed point of $A, B, S$ and $T$. Uniqueness of the fixed point is an easy consequence of inequality (3.2). Hence the result.

Q.E.D.

We now give an example to illustrate the above theorem.

Example 3.2. Let $X=[2,20]$ and for each $t>0$ and $x, y \in X$ define $(X, M, *)$ by $M(x, y, t)=$ $\frac{t}{t+|x-y|}, t>0$ and $M(x, y, 0)=0$. Define self maps $A, B, S$ and $T$ on $X$ by

$A x=2$ if $x=2$ or $>5, A x=x+1$ if $2<x \leq 5$,

$B x=2$ if $x=2$ or $>5, B x=x+2$ if $2<x \leq 5$,

$S 2=2, S x=8$ if $2<x \leq 5, S x=\frac{x+1}{3}$ if $x>5$,

$T x=2$ if $x=2$ or $>5, T x=x+1$ if $2<x \leq 5$.

Then the self maps $A, B, S$ and $T$ satisfy all of the conditions of the above theorem and have a unique common fixed point at $x=2$. Moreover the maps satisfy neither the $\varphi$-contractive condition nor the Banach type contractive condition. Also, one may verify that the self maps $A, B, S$ and $T$ are discontinuous at the common fixed point $x=2$ and $S X$ is a complete subspace of $X$.

Now we shall improve the above theorem using common property (E.A), since it relaxes containment of the range of one map into the range of other, which is utilized to construct the sequence of joint iterates in common fixed point considerations.

Theorem 3.3. Let $A, B, S$ and $T$ be four self maps in a fuzzy metric space $(X, M, *)$, with $a * b$ $=\min \{a, b\}$ for all $a, b \in[0,1]$ satisfying condition $(3.2)$, and

(3.4) the pairs $(A, S)$ and $(B, T)$ satisfy common property $(E . A)$,

(3.5) $S X$ and $T X$ are closed subsets of $X$.

Then

(I) $A$ and $S$ have a coincidence point, 
(II) $B$ and $T$ have a coincidence point.

Moreover, if the pairs $(A, S)$ and $(B, T)$ are weakly compatible, then the maps $A, B, S$ and $T$ have a unique common fixed point in $X$.

Proof. In view of (3.4), there exist two sequences $\left\{x_{n}\right\}$ and $\left\{y_{n}\right\}$ in $X$ such that $\lim _{n \rightarrow \infty} A x_{n}=$ $\lim _{n \rightarrow \infty} S x_{n}=\lim _{n \rightarrow \infty} B y_{n}=\lim _{n \rightarrow \infty} T y_{n}=z$ for some $z \in X$. Since $S X$ is a closed subset of $X$, there exists a point $u \in X$ such that $z=S u$. We claim that $A u=z$. If $A u \neq z$, then, by (3.2) (or equation (3)), take $x=u, y=y_{n}$. Then

$$
M\left(A u, B y_{n}, t\right)>m\left(u, y_{n}, t\right)=\min \left\{M\left(S u, T y_{n}, t\right), M(S u, A u, t), M\left(B y_{n}, T y_{n}, t\right)\right\}
$$

Taking the limit as $n \rightarrow \infty$, we have

$$
M(A u, z, t)>\min \{M(z, z, t), M(z, A u, t), M(z, z, t)\}=M(z, A u, t),
$$

a contradiction. Therefore, $A u=z=S u$, which shows that $u$ is a coincidence point of the pair $(A, S)$.

Since $T X$ is also a closed subset of $X, \lim _{n \rightarrow \infty} T y_{n}=z \in T X$, and hence there exists a $v \in X$ such that $T v=z=A u=S u$. Now we show that $B v=z$.

If $B v \neq z$, then, by using inequality (3.2), take $x=u, y=v$. We then

$$
\begin{gathered}
M(A u, B v, t)>m(u, v, t)=\min \{M(S u, T v, t), M(S u, A u, t), M(B v, T v, t)\}, \\
M(z, B v, t)>\min \{M(z, z, t), M(z, z, t), M(B v, z, t)\}=M(B v, z, t),
\end{gathered}
$$

which is a contradiction. Therefore, $B v=z=T v$, which shows that $v$ is a coincidence point of the pair $(B, T)$.

Since the pairs $(A, S)$ and $(B, T)$ are weakly compatible and $A u=S u, B v=T v, A z=A S u=$ $S A u=S z, B z=B T v=T B v=T z$.

If $A z \neq z$, then, by using inequality (3.2), take $x=z, y=v$ to get

$$
\begin{aligned}
& M(A z, B v, t)>m(z, v, t)=\min \{M(S z, T v, t), M(S z, A z, t), M(B v, T v, t)\}, \\
& M(A z, z, t)>\min \{M(A z, z, t), M(A z, A z, t), M(B v, B v, t)\}=M(A z, z, t),
\end{aligned}
$$

a contradiction. Therefore, $A z=z=S z$.

Similarly, one can prove that $B z=T z=z$. Hence, $A z=B z=S z=T z$, and $z$ is common fixed point of $A, B, S$ and $T$. Uniqueness of the fixed point is an easy consequence of inequality (3.2). Hence the result. We now give an example to illustrate the above theorem.

Q.E.D.

Example 3.4. Let $X=[2,20]$ and for each $t>0$ and $x, y \in X$ define $(X, M, *)$ by $M(x, y, t)=$ $\frac{t}{t+|x-y|}, t>0$, and $M(x, y, 0)=0$. Define self maps $A, B, S$ and $T$ on $X$ by

$A x=2, x=2$ or $>5$,

$A x=x+1$ if $2<x \leq 5$,

$B x=2$ if $x=2$ or $>5, B x=x+2$ if $2<x \leq 5$,

$S x=2$ if $x=2$ or $>5, S x=8$ if $2<x \leq 5$, 
$T x=2$ if $x=2$ or $>5, T x=9$ if $2<x \leq 5$.

Take $\left\{x_{n}=5+\frac{1}{n}\right\}$ and $\left\{y_{n}=5+\frac{1}{n}\right\}$. Then

$$
\lim _{n \rightarrow \infty} A x_{n}=\lim _{n \rightarrow \infty} S x_{n}=\lim _{n \rightarrow \infty} B y_{n}=\lim _{n \rightarrow \infty} T y_{n}=2 \in X
$$

Thus the pairs $(A, S)$ and $(B, T)$ satisfy common property $(E . A)$. One can easily verify that the self maps $A, B, S$ and $T$ satisfy all of the conditions of the above theorem and have a unique common fixed point at $x=2$. Here $S X$ and $T X$ are closed subspaces of $X$ whereas neither $A X$ nor $B X$ is closed subspace of $X$. Moreover, the maps neither satisfy a $\varphi$-contractive condition nor a Banach type contractive condition. Also one may notice that neither $B X \nsubseteq S X$ nor $A X \nsubseteq T X$ and at the common fixed point $x=2$, self maps $A, B, S$ and $T$ are discontinuous.

Finally, it is observed that common property $(E . A)$ requires the completeness or closedness of the subspaces for the existence of the common fixed point. So an attempt has been made to drop the closedness of the subspaces from Theorem 5 by using the $J C L R_{S T}$ property.

Theorem 3.5. Let $A, B, S$ and $T$ be four self maps of a fuzzy metric space $(X, M, *)$, with $a * b$ $=\min \{a, b\}$ for all $a, b \in[0,1]$ satisfying condition (3.2), and

$$
(A, S) \text { and }(B, T) \text { satisfy the } J C L R_{S T} \text { property. }
$$

Then the pairs $(A, S)$ and $(B, T)$ have a coincidence point. Further, if $(A, S)$ and $(B, T)$ are weakly compatible pairs of self maps of $X$, then $A, B, S$ and $T$ have a unique common fixed point in $X$.

Proof. As the pairs $(A, S)$ and $(B, T)$ satisfy the $J C L R_{S T}$ property, there exist two sequences $\left\{x_{n}\right\}$ and $\left\{y_{n}\right\}$ in $X$ such that

$$
\lim _{n \rightarrow \infty} A x_{n}=\lim _{n \rightarrow \infty} S x_{n}=\lim _{n \rightarrow \infty} B y_{n}=\lim _{n \rightarrow \infty} T y_{n}=S u=T u
$$

for some $u \in X$.

First, we assert that $A u=S u$. By (3.2) (or equation (3)), take $x=u, y=y_{n}$ to get

$$
M\left(A u, B y_{n}, t\right)>m\left(u, y_{n}, t\right)=\min \left\{M\left(S u, T y_{n}, t\right), M(S u, A u, t), M\left(B y_{n}, T y_{n}, t\right)\right\} .
$$

Taking the limit as $n \rightarrow \infty$, we have

$$
M(A u, S u, t)>\min \{M(S z, S u, t), M(S u, A u, t), M(S u, S u, t)\}=M(S u, A u, t),
$$

a contradiction. Therefore, $A u=S u$, which shows that $\mathrm{u}$ is a coincidence point of the pair $(A, S)$. Secondly, we assert that $B u=T u$. Using (3.2), take $x=u, y=u$ to get

$$
\begin{gathered}
M(A u, B u, t)>m(u, u, t)=\min \{M(S u, T u, t), M(S u, A u, t), M(B u, T u, t)\}, \\
M(T u, B u, t)>\min \{M(S u, S u, t), M(T u, T u, t), M(B u, T u, t)\}=M(T u, B u, t),
\end{gathered}
$$

a contradiction. Hence $B u=T u$, which shows that $u$ is a coincidence point of the pair $(B, T)$. Thus we have $T u=B u=A u=S u$. 
Now, we assume that $z=T u=B u=A u=S u$. Since the pairs $(A, S)$ and $(B, T)$ are weakly compatible and $A u=S u, B u=T u, A z=A S u=S A u=S z$ and $B z=B T u=T B u=T z$.

If $A z \neq z$, then, by using inequality (3.2), take $x=z, y=u$, to obtain

$$
\begin{gathered}
M(A z, B u, t)>m(z, u, t)=\min \{M(S z, T u, t), M(S z, A z, t), M(B u, T u, t)\} \\
M(A z, z, t)>\min \{M(A z, z, t), M(A z, A z, t), M(z, z, t)\}=M(A z, z, t),
\end{gathered}
$$

a contradiction. Therefore, $A z=z=S z$.

Similarly, one can prove that $B z=T z=z$. Hence $A z=B z=S z=T z$, and $z$ is a common fixed point of $A, B, S$ and $T$. Uniqueness of the fixed point is an easy consequence of inequality (3.2). Hence the result.

Q.E.D.

We now give an example to illustrate the above theorem.

Example 3.6. Let $X=[2,20]$ and for each $t>0$ and $x, y \in X$ define $(X, M, *)$ by $M(x, y, t)=$ $\frac{t}{t+|x-y|}, t>0$ and $M(x, y, 0)=0$. Define self maps $A, B, S$ and $T$ on $X$ by

$A x=2$ if $x=2$ or $>5, A x=x+1$ if $2<x \leq 5$,

$B x=2$ if $x=2$ or $>5, B x=x+2$ if $2<x \leq 5$,

$S x=2$ if $x=2$ or $x>5, S x=x+1$ if $2<x \leq 5$,

$T x=2$ if $x=2$ or $>5, T x=x+9$ if $2<x \leq 5$.

Take $\left\{x_{n}=5+\frac{1}{n}\right\}$ and $\left\{y_{n}=5+\frac{1}{n}\right\}$. Then

$$
\lim _{n \rightarrow \infty} A x_{n}=\lim _{n \rightarrow \infty} S x_{n}=\lim _{n \rightarrow \infty} B y_{n}=\lim _{n \rightarrow \infty} T y_{n}=2=S(2)=T(2) .
$$

Thus the pairs $(A, S)$ and $(B, T)$ satisfy the $J C L R_{S T}$ property. Also the self maps $A, B, S$ and $T$ satisfy all of the conditions of the above theorem and have a unique common fixed point at $x=2$. Notice that none of $A X, B X, S X$ and $T X$ is a closed or complete subspace of $X$. Also, at the common fixed point $x=2$, all of the self maps $A, B, S$ and $T$ are discontinuous. Moreover, the maps satisfy neither the $\varphi$-contractive condition nor the Banach type contractive condition. Also $B(X) \nsubseteq S(X)$ and $A(X) \nsubseteq T(X)$.

\section{References}

[1] M. Akkouchi, A Meir Keeler type common fixed point theorems in four mappings, Opuscula Mathematica, 31(1) (2011), 5-14.

[2] M. Abbas, I. Altun and D. Gopal, Common fixed point theorems for non compatible mappings in fuzzy metric spaces, Bull. Math. Anal. Appl., 1(2) (2009), 47-56.

[3] M. Aamri and D. El Moutawakil, Some new common fixed point theorems under strict contractive conditions, J. Math. Anal. Appl., 270 (2002), 181-188.

[4] S. S. Bhatia, S. Manro and S. Kumar, Fixed point theorem for weakly compatible maps using E.A. property in fuzzy metric spaces satisfying contractive condition of integral type, Int. J. Contemp. Math. Sciences, 5 (51) (2010), 2523-2528. 
[5] S. Chauhan, W. Sintunavarat and P. Kumam, Common Fixed point theorems for weakly compatible mappings in fuzzy metric spaces using (JCLR) property, Applied Mathematics (in press).

[6] Y. J.Cho, Fixed points in fuzzy metric spaces, J. Fuzzy Math., 5 (1997), 949-962.

[7] Y. J. Cho, H. K. Pathak, S. M. Kang and J. S. Jung, Common fixed points of compatible maps of Type (b) on fuzzy metric spaces, Fuzzy Sets and Systems, 93 (1998), 99-111.

[8] A. George and P. Veeramani, On some results in fuzzy metric spaces, Fuzzy Sets and Systems, 64 (1994) 395-399.

[9] A. George and P. Veeramani, On some results of analysis for fuzzy metric spaces, Fuzzy Sets and Systems, 90 (1997), 365-368.

[10] D. Gopal, M. Imdad and C. Vetro, Impact of common property (E.A.) on fixed point theorems in fuzzy metric spaces, Fixed Point Theory and Applications, Volume 2011, Article ID 297360, 14 pages.

[11] S. Heilpern, Fuzzy mappings and fixed point theorems, J. Math. Anal. Appl., 83 (1981), 566569.

[12] M. Imdad and J. Ali, A general fixed point theorem in fuzzy metric spaces via an implicit function, Journal of Applied Mathematics and Informatics, 26 (2008), 591-603.

[13] G. Jungck, Commuting mappings and fixed points, Amer. Math. Mon., 83 (1976), 261-263.

[14] G. Jungck, Compatible mappings and common fixed points, Internat. J. Math. Math. Sci., 9 (1986), 771-779.

[15] I. Kramosil and J. Michalek, Fuzzy metric and statistical spaces, Kybernetica, 11 (1975), 336344.

[16] S. Manro, A common fixed point Theorem for weakly compatible maps satisfying property (E.A) in fuzzy metric spaces using strict contractive condition, ARPN Journal of Science and Technology, 2(4) (2012), 367-370.

[17] S. Manro, S.S. Bhatia and S. Kumar, Common fixed point theorems in fuzzy metric spaces, Annals of Fuzzy Mathematics and Informatics, 3(1)(2012), 151-158.

[18] A. Meir and E. Keeler, A theorem on contraction mappings, J. Math. Anal. Appl., 28 (1969), $326-329$.

[19] K. Menger, Statistical metrics, Proc. Nat. Acad. Sci. (USA), 28 (1942), 535-537.

[20] D. ORegan and M. Abbas, Necessary and sufficient conditions for common fixed point theorems in fuzzy metric spaces, Demonstratio Math., 42(4) (2009), 887-900.

[21] R. P. Pant and P. C. Joshi, A Meir Keeler type fixed point theorem, Indian Journal of Pure and Applied Mathematics, 32(6) (2001), 779-787. 
[22] R. P. Pant and V. Pant, Some fixed point theorem in fuzzy metric space, J. Fuzzy Math, 16(3) (2008), 599-611.

[23] R. P. Pant, A new common fixed point principle, Soochow Journal of Mathematics, 27(3) (2001), 287-297.

[24] V. Pant and K. Jha, $(\varepsilon, \delta)$ contractive condition and common fixed points, Fasciculi Mathematici, 42 (2009), 73-84.

[25] B. Schweizer and A. Sklar, Probabilistic metric paces, North Holland Amsterdam, 1983.

[26] B. Singh and M. S. Chauhan, Common fixed points of compatible maps in fuzzy metric spaces, Fuzzy Sets and Systems, 115 (2000), 471-475.

[27] S. Sessa, On a weak commutativity condition of mappings in fixed point considerations, Publ. Inst.Math., 32(46) (1982), 149-153.

[28] W. Sintunavarat and P. Kumam, Common fixed points for R-weakly commuting in fuzzy metric spaces, Ann Univ Ferrara, DOI 10.1007/s11565-012-0150-z.article ID 637958.

[29] L. A. Zadeh, Fuzzy sets, Infor. and Control., 8 (1965), 338-353. 